\title{
Flexural Behavior of Sandwich Composite Made of JFRP Honeycomb as Core and GFRP as Skin
}

\author{
Md. Rakibul Islam, Md. Arifuzzaman*, Asif Karim Neon, Md. Shahe Duzzaman and Md. Rafiul Islam \\ Department of Mechanical Engineering, Khulna University of Engineering \& Technology, Khulna-9203, BANGLADESH
}

Received: September 23, 2020, Revised: November 21, 2020, Accepted: November 25, 2020, Available Online: November 29, 2020

\begin{abstract}
The increasing demand of lightweight, strong and sustainable materials in aerospace, automobile and marine sectors is leading towards the development of new materials and structures. The sandwich composite is one of them which is well-known for their high strength to weight ratio and the fiber based sandwich structures with cellular core show comparatively good mechanical, acoustic, thermal and energy absorption properties than metallic cellular structure. The purpose of this work is to fabricate a sandwich structure with jute fiber reinforced polymer composite (JFRP) as core and glass fiber reinforced polymer composite (GFRP) as face sheet and to investigate bending properties of the fabricated structures for varying face sheet thicknesses. Skin and core honeycomb strips of the sandwich composites were manufactured using hand layup method and steel mold was used to obtain honeycomb shape. Flexural test results show that face sheet thickness has significant effect on the flexural behavior such as peak load, flexural strength and energy absorption. The failure mechanism during bending tests were also identified which would serve as a basis for future improvement of manufactured composites. The delamination at the interface between the core and the face sheet was the first catastrophic failure during bending. The presented sandwich structures are able to carry a significant amount of load even after failure.
\end{abstract}

Keywords: Sandwich composite; JFRP; GFRP; Honeycomb; Flexural properties; Energy absorption.

This work is licensed under a Creative Commons Attribution-Non Commercial 4.0 International

\section{Introduction}

A sandwich composite structure is constructed by fastening two thin but resilient face sheets to a thick but lightweight core. Core materials provide high flexural stiffness with overall low density the composite material. The separation of thin face sheets with a light weight core significantly increase the second moment of area thus the flexural stiffness of the material cross section increases through a small increase in weight. Various types of cores are used in sandwich structures i.e. honeycomb, corrugated, cellular etc. By varying the core types, geometry, thickness and material it is possible to achieve a wide range of properties and anticipated performances. Now a days honeycomb sandwich structures are widely used in damping and weight sensitive structures. In automobile, marine and aerospace industries, these materials are being used extensively due to their excellent weight saving characteristics without compromising the strength [1]. In a honeycomb sandwich structure, flexural loads are carried by forming force couple by the face sheets and shear loads are carried by lightweight core materials [2]. So, the honeycomb sandwich panels are highly efficient for carrying bending loads. Under flexural loading, one laminate is subjected to compression and the other one is subjected to tension which forming a force couple as face sheets acts together [3]. Many researchers have worked to develop honeycomb core based sandwich structure using either metals or fiber reinforced polymer composites. However, the composite honeycomb performs much better than metallic honeycombs under both quasi static and dynamic condition although the cost of composite honeycomb was higher than metallic honeycombs [4]. Thomas et al. [4] studied the influence of cell wall thickness, node length, cell size and loading on the bending and crushing responses of honeycomb structure in both out-of-plane and in- plane loading conditions. They concluded that the geometrical core configuration and geometric property like cell size, cell wall thickness and node length etc. play a crucial role in administrating potential of honeycomb. Thomas et. al. [4] also found that the face sheet material and their thickness affects the energy absorption capability. Hussain et al. [5] explored the static failure behavior of wood based sandwich panels using woodbased core and face sheets made of glass fiber reinforced polymer. It was found that, the load carrying ability of sandwich structures with plywood and solid MDF cores was higher compared to sandwich structures with honeycomb cores, but honeycomb sandwich panels can carry significant amount of loads even after the failure. The hexagonal honeycomb core shape has high strength and load carrying capacity along with minimum material and space requirement [4]. Most of the cellular sandwich structures are made of metals or polymers because of the complexity of manufacturing cellular cores using fiber reinforced composite. For core material using natural fiber like jute is cheaper, eco-friendly, lower mass per unit area, biodegradable, recyclable and offer good acoustic insulating properties as well [6]. As face sheet material glass fiber reinforced composites show good mechanical performance and water resistance properties [7], [8]. A combination of natural and synthetic fibers may provide an improved performance as a hybrid sandwich structure.

Therefore, in this paper a hybrid sandwich composite is introduced in which the natural jute fiber is used to fabricate the honeycomb core and the synthetic glass fiber reinforced epoxy as a face sheet. The manufacturing and flexural behavior of the fabricated sandwich structure is investigated in terms of peak load, bending strength, interfacial shear stress, energy absorption and failure mechanism. 


\section{Experimental details}

\subsection{Constituent materials}

The constituent materials used for manufacturing the sandwich composites are E-glass fiber mat, jute fiber mat, and epoxy resin with hardener. E-glass fiber mat of $0.45 \mathrm{~mm}$ thickness was used as reinforcement on epoxy to manufacture sandwich face sheet. Jute fiber mat of $0.85 \mathrm{~mm}$ thickness was used in epoxy matrix for making honeycomb core strips.

\subsection{Specimen manufacturing}

\subsubsection{Mold making}

A steel mold is modelled and manufactured in CNC machine to provide the required shape of the jute fiber reinforced epoxy composite strips for making honeycomb core. The CAD model of the mold is given in Fig. 1.

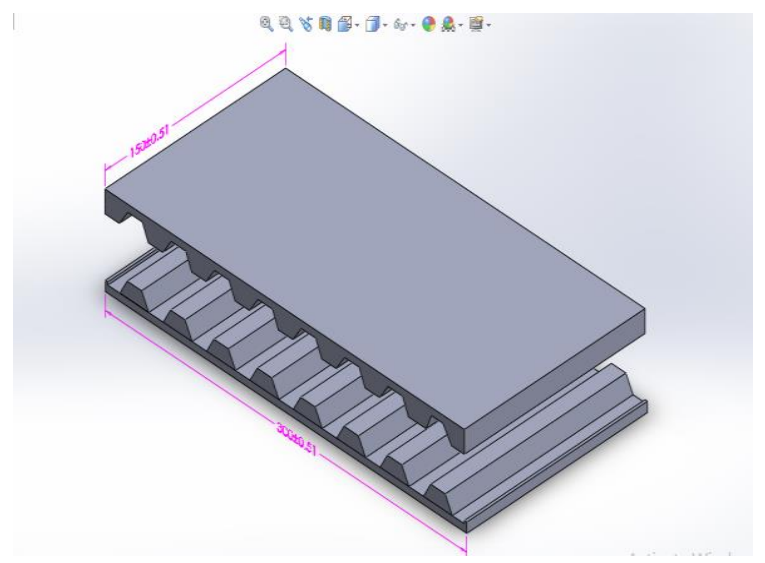

Fig. 1 CAD model of the mold with dimensions $300 \mathrm{~mm} \times 150$ $\mathrm{mm}$

\subsubsection{Face sheet making}

At first, the resin and hardener was blended in a pot according to the ratio of resin to hardener (10:1) provided by the manufacturer. On top of a plastic sheet a layer of epoxy was placed first and the reinforcing glass fiber mat was laid then another layer of epoxy was poured over the mat. Then the layup has been rolled with a wooden roller in order to remove any bubbles and to ensure proper epoxy distribution. The layup was kept between two metal plates with approximately $10 \mathrm{~kg}$ weight of top metal plate and cured for 24 hours. Similar method was adopted to manufacture double and triple layer face sheets. Finally the manufactured face sheets were cut to the required size.

\subsubsection{Core strip making}

At first, the resin and hardener was mixed at 10:1 ratio given by the manufacturer. Then the jute fiber mat was wetted by the epoxy using a hand layup method. The layup was kept on top of the lower mold part and the upper part of the mold was placed. The whole setup was kept under pressure with a $10 \mathrm{~kg}$ load on the top plate for 24 hours for curing. The desired size of the core strips was cut from the manufactured panel.

\subsubsection{Sandwich preparation}

The core of the sandwich structure was made by joining the core strips together using epoxy adhesive in such a way that they form hexagonal cells as shown in Fig. 2 (a). The face sheets were then attached (see Fig. 2(b)) with the core using epoxy to obtain the finished composite beams (see Fig. 2(c)). The width of the bending specimens were $b=46.5 \mathrm{~mm}$ for all samples.

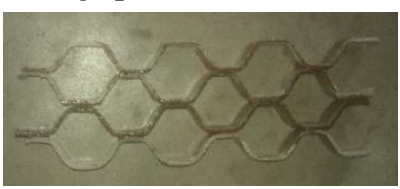

(a)

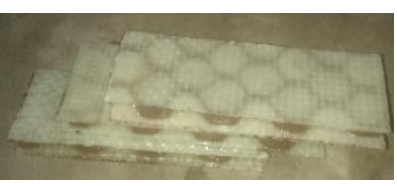

(c)

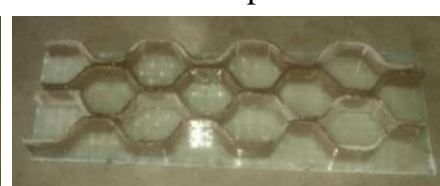

(b)

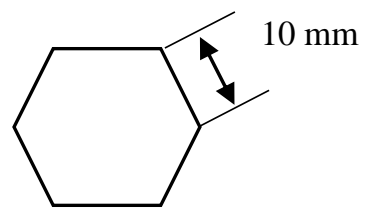

(d)
Fig. 2 (a) The hexagonal honeycomb core made using core strips; (b) the core was attached with face sheet; (c) the fabricated sandwich structure; and (d) schematic diagram of cell size.

\subsection{Mechanical tests}

Flexural test was conducted in the Universal Testing Machine (UTM) with digital data acquisition system for the load and the displacements at a cross head speed of approximately $5 \mathrm{~mm} / \mathrm{min}$. At least three specimens for each category were tested to obtain the average values of flexural properties. International standard ASTM D790-03 [9] and ASTM C393/C393M-11 [10] were used as a guide for flexural test and the calculation of bending properties.

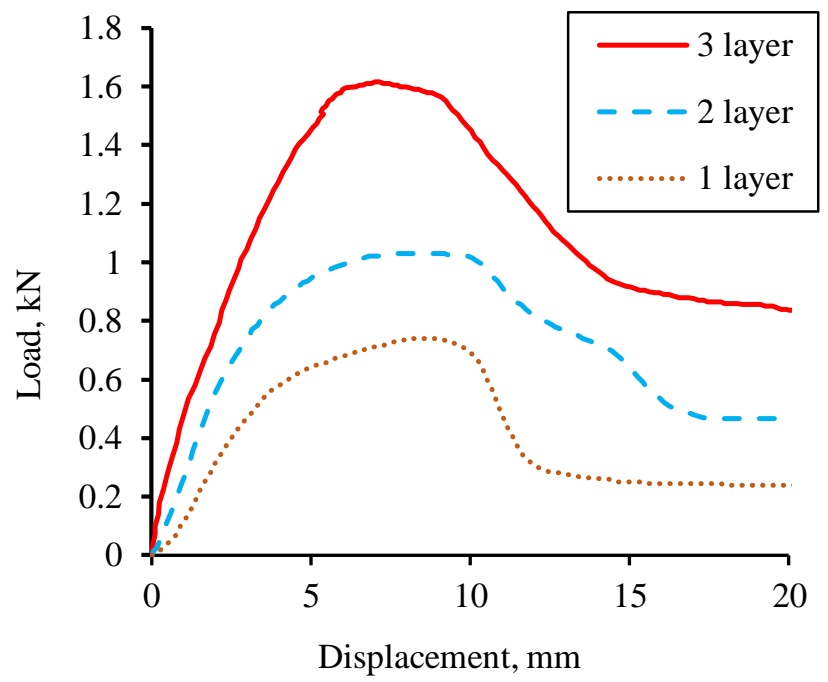

Fig. 3 Typical load - displacement curves for sandwich composite under three point bending for 1,2 and 3 layers of glass fiber mat in the face sheet.

\section{Results and discussion}

\subsection{Flexural properties}

Typical load-displacement curves obtained from three point bend test of three different sandwich composites with different layers in the face sheet (i.e. 1, 2 and 3 layers of glass fiber mats in the face sheet) are given in Fig. 3. All curves show similar trends i.e. the load increases linearly with the displacement then 
the rate of increase of load with displacement starts to decrease at some point and a pseudo-plateau region is seen. After that load starts to decrease gradually until another plateau is noticed. The failure of the composite actually starts and completes in the pseudo-plateau region. It can also be observed from Fig. 3 that the linear elastic line becomes stiffer for the higher thickness of the face sheet but the trend of the load-displacement curve remains the same.

The peak load and the bending strength with the mass of fabricated sandwich composite are plotted in Fig. 4 for three different face sheet thicknesses with standard deviations as error bar. The peak load and the bending strength both increased with increasing face sheet thickness. Sandwich structures reported in literature also revealed similar observations [11]-[12][14]. However the increase in peak load/bending strength is obtained with the cost of the weight of the composites.

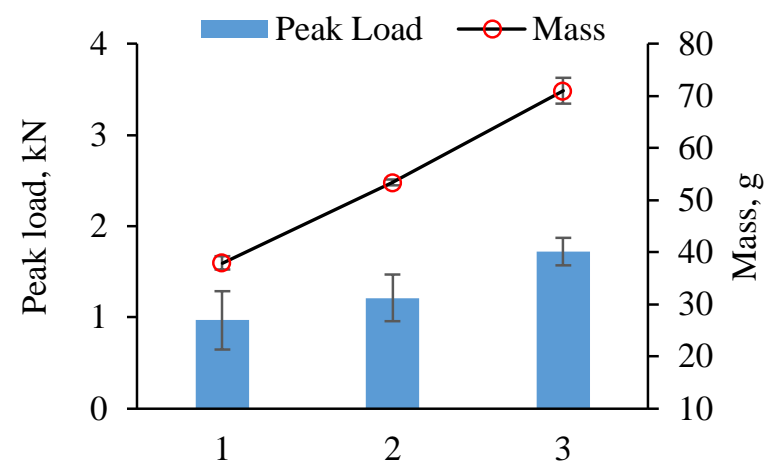

The number of glass fiber layers on face sheets

(a)

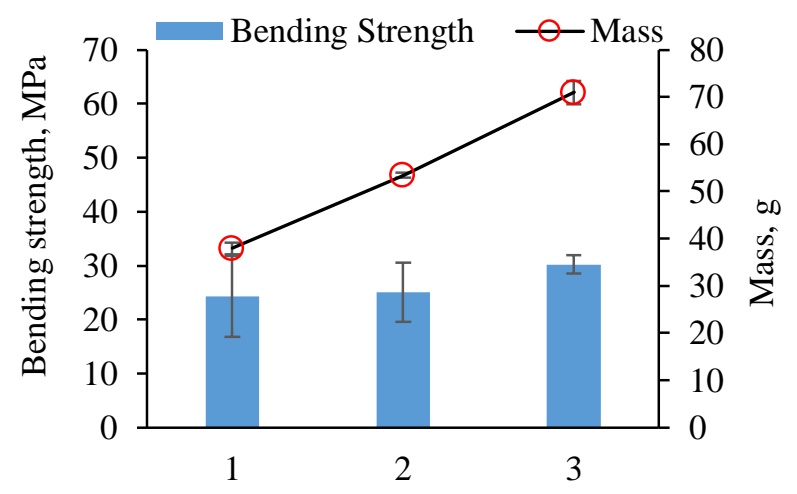

The number of glass fiber layers on face sheets

(b)

Fig. 4 (a) Peak load and (b) bending strength as a function of the number of glass fiber layers in the face sheet.

When the glass fiber layer in the face sheet was increased to two the peak load and bending strength were increased by $25.32 \%$ and $3.29 \%$ respectively but the mass of sandwich structure was increased by $40.61 \%$. In the case of three layer glass fiber in the face sheet the peak load and bending strength was increased by $77.48 \%$ and $24.58 \%$ while the mass was increased by $86.91 \%$. The specific strength is a key indicator for a material to be assumed as a lightweight material. The specific bending strength of the manufactured sandwich structures were found to be 639.13, 469.51 and $426.00 \mathrm{MPa} / \mathrm{g}$ for 1 layer, 2 layers and 3 layers of glass fiber in the face sheet respectively. This clearly shows that the thicker face sheet makes the sandwich structure heavier with higher bending strength.

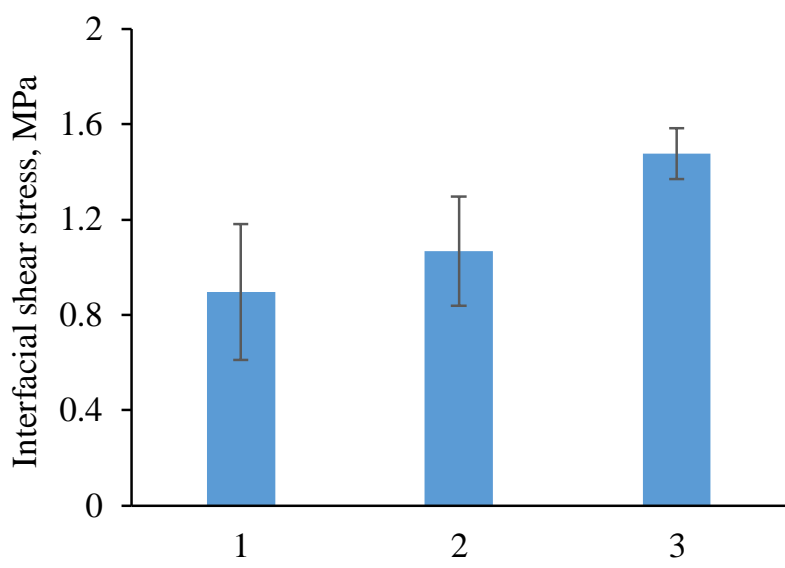

The number of glass fiber layers in the face sheets

Fig. 5 Shear stress at the core/face sheet interface as a function of the number of glass fiber layers in the face sheet.

The shear stress calculated at the core/face sheet interface is given in Fig. 5 for various face sheets used in this study. It is seen that the shear stress also increased with increasing the number of glass fiber layers in the sandwich skin alike the peak load and the bending strength. The interfacial shear stress $=\frac{P}{(d+c) * b}$ where, $\mathrm{P}$, $\mathrm{d}, \mathrm{c}$ and $\mathrm{b}$ are the peak load, sandwich height, core height and beam width of the sandwich structure [10]. According to this formula, the shear stress at the interface between the skin and the core increases with the increase in peak load but decreases with the increase in sandwich height. When the number of layer was increased in the face sheet without changing the core height there is a slight increase in sandwich height but the peak load increased considerably as shown in Fig. 4 (a) and this is the reason for the increase in shear stress in the interface between the core and skin when the face sheet thickness increased. The shear stress was increased by $19.16 \%$ and $64.67 \%$ when the number of layers in the face sheet was increased to two and three respectively.

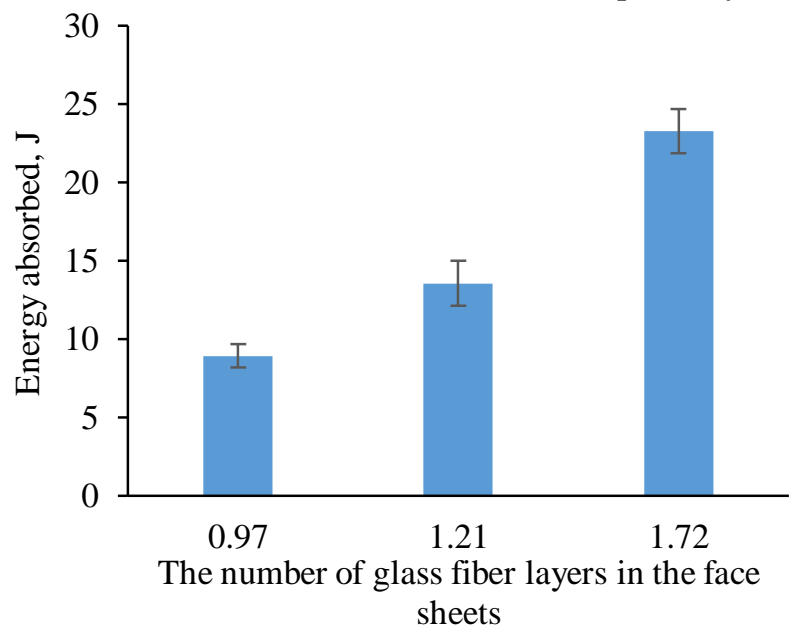

Fig. 6 Energy absorbed by the sandwich composite for a cross head displacement of $20 \mathrm{~mm}$. 
The energy absorbed by the sandwich structure during bending was calculated from the area under the loaddisplacement curve up to a cross head displacement of $20 \mathrm{~mm}$. The energy absorption is plotted for various face sheet thicknesses in Fig. 6. The bending energy absorption increased with increasing the face sheet thickness as expected. Since the load bearing capacity was increased with the increase in face sheet thickness, the energy absorption must increase with increasing face sheet thickness. From Fig. 3 , it is seen that the load-displacement curves are stiffer for the thicker face sheet thickness and the plateau region is also higher for thicker face sheets. Again the energy absorption is the area under the curve of load-displacement. Therefore the energy absorption must increase with the increase in face sheet thickness. The energy absorption during bending was increased by $51.84 \%$ and $160.73 \%$ when the number of glass fiber layers increased to two and three respectively. The specific energy absorption is found to be $0.23,0.25$ and $0.33 \mathrm{~J} / \mathrm{g}$ for the number of glass fiber layers in face sheet 1,2 and 3 respectively. The specific energy absorption increased with increasing face sheet thickness which indicates that the thicker face sheet provides not only higher strength but also better energy absorption. However this is not always the case as reported by Yan et. al. [14] that the density of core material plays an important role. They have found that for high density foam core the energy absorption capacity of the sandwich decreases with the increase in face sheet thickness.

\subsection{Failure Mechanism}

The photographs of failed specimens and the load versus displacement curves are given in Fig. 7, Fig. 8 and Fig. 9 for sandwich specimens made with a single, double and triple layers of glass fiber mats respectively. For the specimens with a single layer face sheet, it is observed that the delamination started on the top side but either left or right side of the middle load pin. The whole face sheet was not delaminated but a small portion as shown in Fig. 7 (a). The delamination continued until the load became steady again indicating a flat line. The flat line of the load versus displacement curve represents the load carrying capacity of sandwich after failure with the contribution from the lower face sheet and honeycomb core together. In the case of specimens with the double and triple layer face sheets the whole top face sheet was delaminated in one side of the middle load pin as shown in Fig. 8 (a) and Fig. 9 (a) respectively. The load carrying capacity of the sandwich after failure is also seen in the case of specimens with double and triple layer face sheets. No failure has been noticed in both face sheets for the cross head displacement studied in the case of double and triple layer face sheets. But the buckling of the delaminated top face sheet in the case of single layer face sheet specimens was noticed. The face sheet delamination is a very common type of failure for sandwich structures because of the weak bonding between the face sheet and the core. A clear difference in delamination behavior between thin and thick face sheets is seen in this study. That is the thick face sheet causes catastrophic delamination while the thin face sheet causes localized delamination.

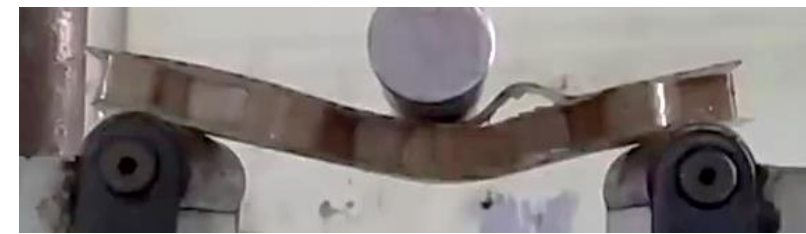

(a)

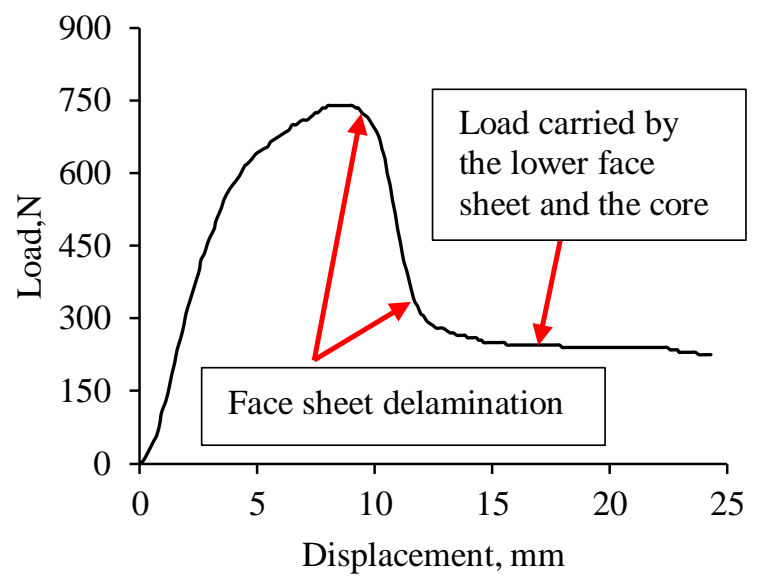

(b)

Fig. 7 A photograph of face sheet delamination and (b) a typical load - displacement curve for specimens with face sheet made of single layer of glass fiber mat.

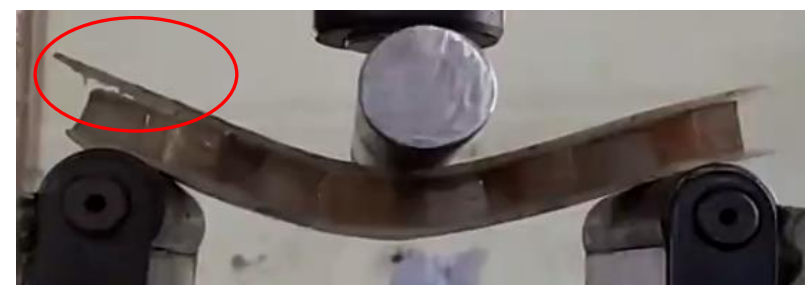

(a)

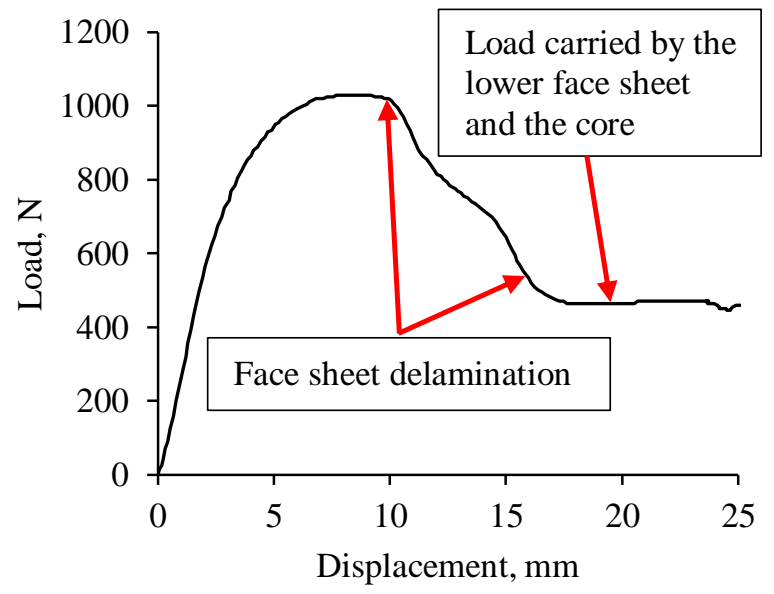

(b)

Fig. 8 (a) A photograph of face sheet delamination and (b) a typical load - displacement curve for specimens with face sheet made of double layer of glass fiber mats. 


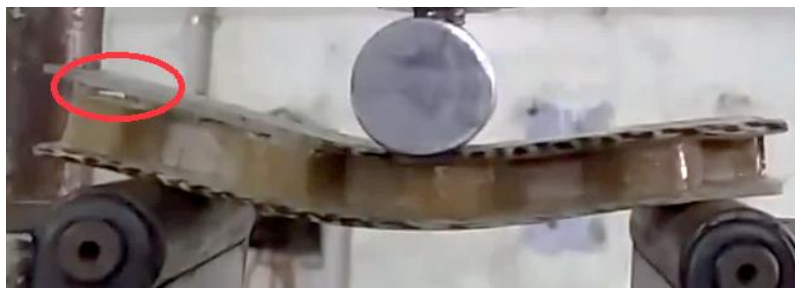

(a)

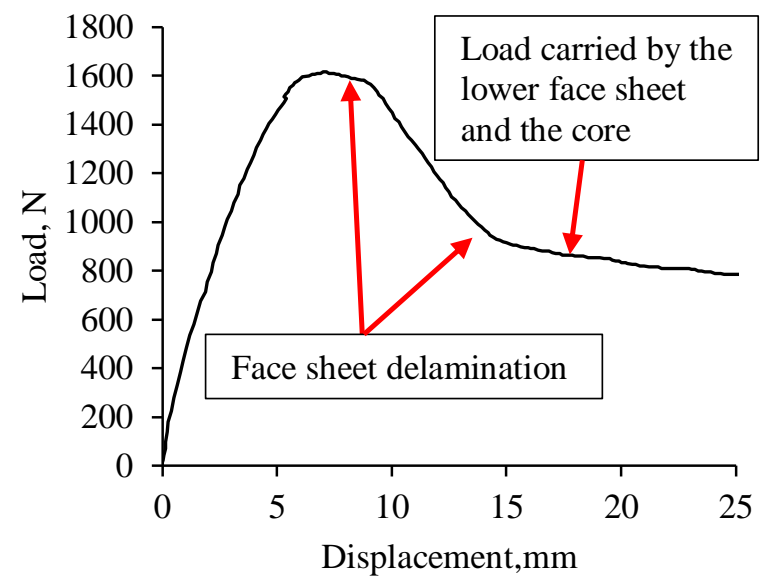

(b)

Fig. 9 A photograph of face sheet delamination and (b) a typical load - displacement curve for specimens with face sheet made of triple layer of glass fiber mats.

\section{Conclusions}

In this work, the sandwich composites with glass fiber/epoxy face sheet and jute fiber/epoxy honeycomb core were fabricated. Three point bending tests were conducted to investigate the bending properties and failure mechanism of the fabricated structure for various face sheet thicknesses. It was observed that face sheet thickness plays an important role on the flexural properties of the manufactured honeycomb sandwich structure. The peak load, flexural strength and core shear stress and energy absorption increased with increasing the thickness of face sheets but keeping the core height constant. All specimens showed failure due to the delamination of the upper face sheet during flexural tests and it is also observed that the fabricated sandwich structure is capable of carrying a substantial amount of load after failure. The load carrying capacity after failure is higher for thicker face sheets.

\section{Acknowledgements}

Authors acknowledge the partial funding and laboratory facilities provided for this work by Khulna University of Engineering \& Technology.

\section{References}

[1] Yang, Y., Li, B., Chen, Z., Sui, N., Chen, Z., Saeed, M.U., Li, Y., Fu, R., Wu, C. and Jing, Y., 2016. Acoustic properties of glass fiber assembly-filled honeycomb sandwich panels. Composites Part B: Engineering, 96, pp.281-286.

[2] Nguyen, M.Q., Jacombs, S.S., Thomson, R.S., Hachenberg, D. and Scott, M.L., 2005. Simulation of impact on sandwich structures. Composite structures, 67(2), pp.217-227.

[3] Ali, M.X.M., 2016. Performance of Honeycomb Sandwich Structure with Natural Fibres Fabric Reinforced Facesheets (Doctoral dissertation, Universiti Teknologi Malaysia).

[4] Thomas, T. and Tiwari, G., 2019. Crushing behavior of honeycomb structure: a review. International Journal of Crashworthiness, 24(5), pp.555-579.

[5] Hussain, M., Abbas, N., Zahra, N., Sajjad, U. and Awan, M.B., 2019. Investigating the performance of GFRP/wood-based honeycomb sandwich panels for sustainable prefab building construction. SN Applied Sciences, 1(8), p.875.

[6] Du, Y., Yan, N. and Kortschot, M.T., 2012. Light-weight honeycomb core sandwich panels containing biofiberreinforced thermoset polymer composite skins: Fabrication and evaluation. Composites Part B: Engineering, 43(7), pp.2875-2882.

[7] Wambua, P., Ivens, J. and Verpoest, I., 2003. Natural fibres: can they replace glass in fibre reinforced plastics?. Composites science and technology, 63(9), pp.1259-1264.

[8] Mohammed R., Ahmed A., Elgalib M. A., Ali A. H. M., Ahmed H. A., 2016. Damage Behaviors of Glass Fabric Honeycomb Sandwich Composite Materials Under Static Loading. International Journal of Mechanical And Production Engineering, 4(5), pp. 35-40,.

[9] ASTM D 790-03, "Standard Test Methods for Flexural Properties of Unreinforced and Reinforced Plastic and Electrical Insulating Material," PA, USA.

[10] ASTM C393/C393M-11, "Standard Test Method for Core Shear Properties of Sandwich Construction by Beam Flexure," PA, USA, 2012.

[11] Zu, G.Y., Lu, R.H., Li, X.B., Zhong, Z.Y., HAN, M.B. and YAO, G.C., 2013. Three-point bending behavior of aluminum foam sandwich with steel panel. Transactions of Nonferrous Metals Society of China, 23(9), pp.24912495.

[12] Wang, N.Z., Xiang, C.H.E.N., Ao, L.I., Li, Y.X., Zhang, H.W. and Yuan, L.I.U., 2016. Three-point bending performance of a new aluminum foam composite structure. Transactions of Nonferrous Metals Society of China, 26(2), pp.359-368.

[13] Zu, G., Song, B., Zhong, Z., Li, X., Mu, Y. and Yao, G., 2012. Static three-point bending behavior of aluminum foam sandwich. Journal of alloys and compounds, 540, pp.275-278.

[14] Yan, C., Song, X., Zhu, H., Jing, C. and Feng, S., 2018. Flexural response of carbon fiber reinforced aluminum foam sandwich. Journal of Composite Materials, 52(14), pp.1887-1897. 